Technical Report No. 32-427

\title{
Phase-Locked Loop Dynamics in the Presence of Noise by Fokker-Planck Techniques
}

\author{
A. J. Viterbi
}

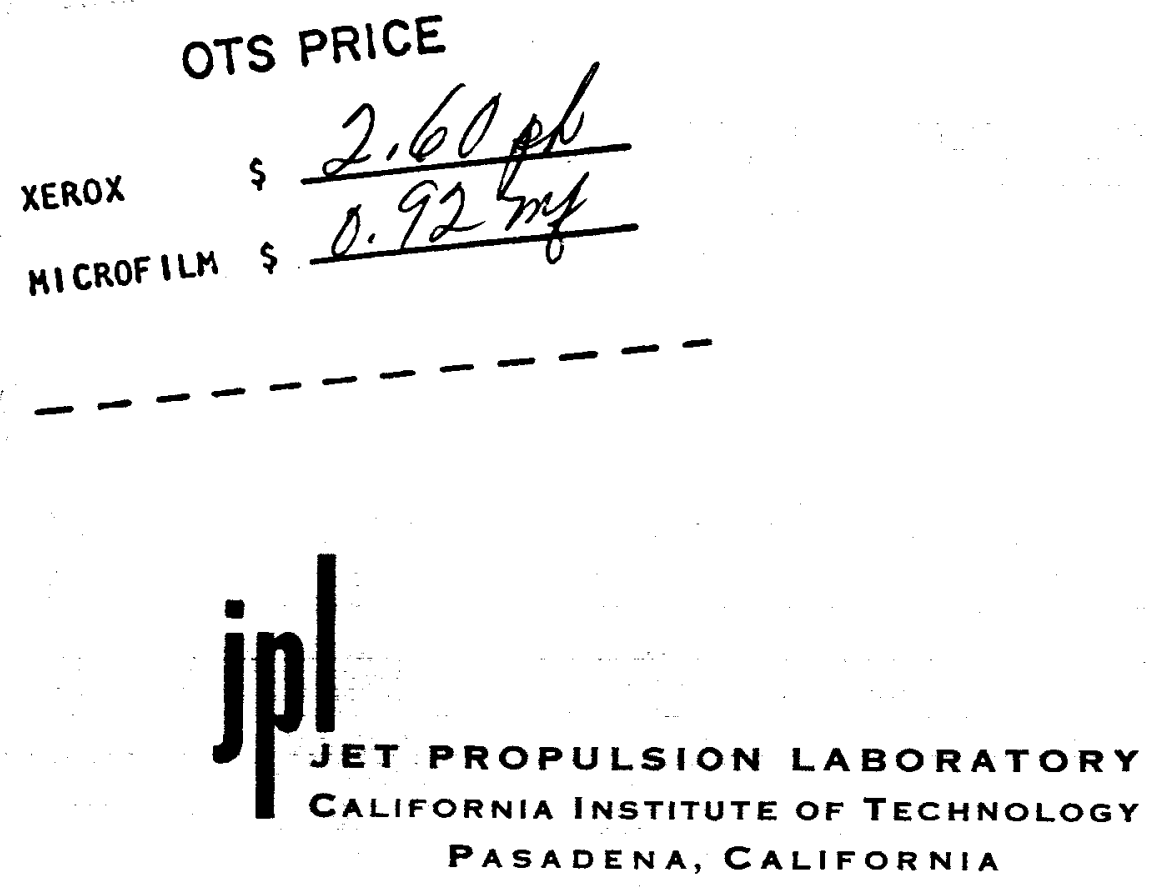

March 29, 1963 
National aeronautics and Space Administration

CONTRACT NO. NAS 7-100

Technical Report No. 32-427

\title{
Phase-Locked Loop Dynamics in the Presence of Noise by Fokker-Planck Techniques
}

\author{
A. J. Viterbi
}

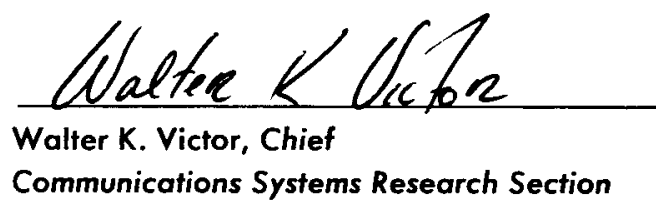

\footnotetext{
JET PROPULSION LABORATORY CALIFORNIA INSTITUTE OF TECHNOLOGY

PASADENA. CaLIFORNIA
}

March 29, 1963 
Copyright (C) 1963

Jet Propulsion Laboratory

California Institute of Technology 


\section{CONTENTS}

I. Introduction . . . . . . . . . . . . . . . . . . . . . 1

II. The First-Order Loop and lts Mechanical Analog . . . . . . 3

III. The Steady-State Phase-Error Probability Density for the First-Order Loop . . . . . . . . . . . . . . . . 5

IV. The Fokker-Planck Equation for Higher-Order Loops . . . . 10

V. Steady-State Probability Distribution for the Second-Order Loop . . . . . . . . . . . . . . . . . . 12

VI. Mean Time to Loss of Lock and Frequency of Skipping Cycles . . . . . . . . . . . . . . . . . . . 14

VII. Threshold Considerations and Conclusions . . . . . . . . 17

References . . . . . . . . . . . . . . . 20

\section{FIGURES}

1. Phase-locked loop . . . . . . . . . . . . . . . . . . . . . 1

2. Mechanical analog of the first-order loop . . . . . . . . . . . 4

3. Qualitative behavior of the probability density function for the first-order loop, $\omega=\omega_{0}$. . . . . . . . . . . . . . . 4

4. Model of first-order loop . . . . . . . . . . . . . . . . . . . . . 6

5. First-order loop, steady-stafe probability densities for $\omega=\omega_{0}$. . . . 7

6. Steady-state, cumulative probability distribution of first-order loop for $\omega=\omega_{0}$. . . . . . . . . . . . . . . . . . 8

7. Variance of phase-error for first-order loop where $\omega=\omega_{0}$. . . . . . 9

8. First-order loop, steady-state probability densities for $\left(\omega=\omega_{0}\right) /(A K)=\sin (\pi / 4)$. . . . . . . . . . . . . . . . . . . . . 9

9. Domain of integral $\mathrm{T}$. . . . . . . . . . . . . . . . . . . . . 15

10. Frequency of skipping cycles normalized by loop bandwidth for first-order loop where $\omega=\omega_{0}$. . . . . . . . . . . . . . 16

11. Domains of integration for $T(\pi)$ and $T^{\prime}(\pi)$. . . . . . . . . . . . . 17

12. Comparison of variance for first-order loop with results of approximate models . . . . . . . . . . . . . . . . . 18 


\section{ABSTRACT}

$$
14881
$$

Statistical parameters of the phase-error behavior of a phase-locked loop tracking a constant frequency signal in the presence of additive, stationary, Gaussian noise are obtained by treating the problem as a continuous random walk with a sinusoidal restoring force. The FokkerPlanck or diffusion equation is obtained for a general loop. An exact expression for the steady-state phase-error distribution is available only for the first-order loop, but approximate and asymptotic expressions are derived for the second-order loop. Results are obtained also for the expected time to loss of lock and for the frequency of skipping cycles. Threshold criteria for the phase-locked loop are discussed, and thresholds of approximate models which have been widely accepted are obtained by comparison with the exact results available for the first-order loop.

\section{INTRODUCTION}

The phase-locked loop is a communication receiver which operates as a coherent detector by continuously correcting its local oscillator frequency according to a measurement of the phase error. A block diagram of the device is shown in Fig. 1 with the pertinent input and output signals indicated. The output of the voltage-controlled oscillator (VCO) is a sinusoid whose frequency is controlled by the input voltage $e(t)$; that is,

$$
\dot{\theta}_{2}(t)=\frac{d \theta_{2}}{d t}=K_{2} e(t)
$$

so that when $e(t)=0$, the oscillator frequency is $\omega_{0}$. The received signal is a sinusoid of power $A^{2}$ w, of arbitrary

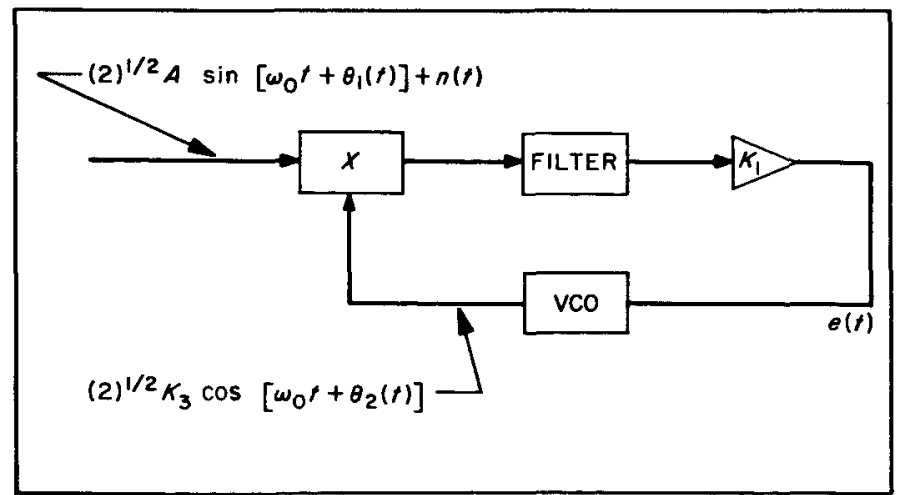

Fig. 1. Phase-locked loop 
frequency $\omega$, and of phase $\theta$. Thus, it is represented by the expression

$$
(2)^{1 / 2} A \sin [\omega t+\theta]=(2)^{1 / 2} A \sin \left[\omega_{0} t+\theta_{1}(t)\right]
$$

where

$$
\theta_{1}(t)=\left(\omega-\omega_{0}\right) t+\theta
$$

The noise is assumed to be stationary, Gaussian, and white of one-sided spectral density $N_{0} \mathrm{w} / \mathrm{cps}$. The noise process over an arbitrary period of duration $T$ can be expanded in a Fourier series whose coefficients become independent Gaussian variables in the limit as $T$ approaches infinity (Ref. 1). By collecting the sine and cosine terms of the series, we can represent the noise process of infinite duration by the expression

$$
n(t)=(2)^{1 / 2} n_{1}(t) \sin (\omega t+\theta)+(2)^{3 / 2} n_{2}(t) \cos (\omega t+\theta)
$$

where $n_{1}(t)$ and $n_{2}(t)$ are both stationary, white, Gaussian processes of one-sided spectral density $N_{0} \mathrm{w} / \mathrm{cps}$ and are statistically independent of one another.

Thus the product of input and reference signals is

$$
\begin{aligned}
2\{A & \sin \left[\omega_{0} t+\theta_{1}(t)\right]+n_{1}(t) \sin (\omega t+\theta) \\
& \left.+n_{2}(t) \cos (\omega t+\theta)\right\}\left\{K_{3} \cos \left[\omega_{0} t+\theta_{2}(t)\right]\right\} \\
= & A K_{3} \sin \left[\theta_{1}(t)-\theta_{2}(t)\right] \\
& +K_{3} n_{1}(t) \sin \left[\left(\omega-\omega_{0}\right) t+\theta-\theta_{2}(t)\right] \\
& +K_{3} n_{2}(t) \cos \left[\left(\omega-\omega_{0}\right) t+\theta-\theta_{2}(t)\right] \\
& + \text { double frequency terms } \\
= & A K_{3} \sin \left[\theta_{1}(t)-\theta_{2}(t)\right] \\
& +K_{3} n_{1}(t) \sin \left[\theta_{1}(t)-\theta_{2}(t)\right] \\
& +K_{3} n_{2}(t) \cos \left[\theta_{1}(t)-\theta_{2}(t)\right] \\
& + \text { double frequency terms }
\end{aligned}
$$

where $\theta_{1}(t)$ is given by Eq. (2). The double frequency terms may be neglected since neither the filter nor the VCO will respond significantly to these for reasonably large $\omega_{0}$. Then from Fig. 1 we see that

$$
\begin{aligned}
e(t)= & K_{1} F(s)\left\{A K_{3} \sin \left[\theta_{1}(t)-\theta_{2}(t)\right]\right. \\
& +K_{3} n_{1}(t) \sin \left[\theta_{1}(t)-\theta_{2}(t)\right] \\
& \left.+K_{3} n_{2}(t) \cos \left[\theta_{1}(t)-\theta_{2}(t)\right]\right\}
\end{aligned}
$$

where $F(s)$ is a rational function which represents in operational notation the effect of the linear filter in the loop. If we let $\phi(t)=\theta_{1}(t)-\theta_{2}(t)$ and $K=K_{1} K_{2} K_{3}$ and use Eq. (1), we obtain

$$
\dot{\phi}(t)=\dot{\theta}_{1}(t)-K_{2} e(t)
$$

Then from Eq. (2) and (3) we have

$$
\begin{aligned}
\dot{\phi}(t)= & \left(\omega-\omega_{0}\right)-K F(s)[A \sin \phi(t) \\
& \left.+n_{1}(t) \sin \phi(t)+n_{2}(t) \cos \phi(t)\right]
\end{aligned}
$$

The instantaneous phase error or difference between the received signal and the reference signal at the output of the VCO is $\phi(t)$. Equation (4) is the exact expression for the operation of the phase-locked loop in the presence of noise. Several authors beginning with Gruen (Ref. 2) have obtained solutions of this equation in the absence of noise for a number of filter transfer functions and also for the case of linearly time-varying input frequency. The most complete treatment of the noise-free performance is contained in Ref. 3. The general case in which additive noise is present has been treated by a variety of approximations. Jaffe and Rechtin (Ref. 4) assumed $\phi(t)$ to be at all times small enough that $\cos \phi \simeq 1$ and $\sin \phi \simeq \phi<<1$ so that the expression in brackets in Eq. (4) becomes $A \phi(t)+n_{2}(t)$. This produces a linear time-invariant model of the system. Recently Van Trees (Ref. 5) refined this analysis by linearizing about the equilibrium point $\phi_{0}$, making the assumption

and

$$
\sin \left(\phi-\phi_{0}\right) \simeq \phi-\phi_{0}
$$

$$
\cos \left(\phi-\phi_{0}\right) \simeq 1
$$

This generates a linear time-varying model. Develet (Ref. 6) applied Booton's quasi-linearization technique (Ref. 7), replacing the sinusoidal nonlinearity by its average gain. Both Van Trees' and Devlet's methods obtain estimates of the noise threshold of the device. Margolis (Ref. 8) obtained a series representation for the moments of the phase error, but the method was too involved to give useful results.

Unlike these analyses, continuous random walk or Fokker-Planck techniques yield exact expressions for the statistics of the random process $\phi(t)$. Unfortunately, expressions in closed form can be obtained only for the first-order loop (i.e., when the filter is omitted). For the general case, a partial differential equation in $\phi$ and its time derivatives is derived, but solutions cannot be obtained in general.

These techniques were first applied to this problem in the Soviet literature by Tikhonov (Ref. 9, 10), who obtained the steady-state probability distribution of $\phi$ 
for the first-order loop enclosed form and an approximate expression for the distribution when the loop contains a one-stage RC filter. Tikhonov's result on the steady-state distribution for the first-order loop is contained in Part III of this Report. The variance and cumulative distribution are also obtained. In Part IV, we derive the Fokker-Planck equation for the general loop filter which produces zero mean error. In Part V, this equation is specialized to the second-order loop, and the form of the solution for the steady-state probability distribution of $\phi$ is obtained. Part VI presents results on the mean time to loss of lock and the frequency of skipping cycles for the first-order loop, which is a random walk problem with absorbing boundaries. Finally, in Part VIII, the results are compared with those of the above mentioned approximate models in an attempt to determine validity thresholds for the models and a performance threshold for the device.

First of all, in the next Part, a simple mechanical analog of the phase-locked loop is presented which provides a qualitative description of the operation of the device and an understanding of the nature of the statistical parameters required for its quantitative description.

\section{THE FIRST-ORDER LOOP AND ITS MECHANICAL ANALOG}

If the filter is omitted, we let $F(s)=1$ in Eq. (4) and obtain the first-order differential equation

$$
\begin{aligned}
\dot{\phi}(t)= & \left(\omega-\omega_{0}\right)-K[A \sin \phi(t) \\
& \left.+n_{1}(t) \sin \phi(t)+n_{2}(t) \cos \phi(t)\right]
\end{aligned}
$$

Hence the term "first-order" loop. Since $n_{1}(t)$ and $n_{2}(t)$ are both white, the instantaneous change in $\phi$ represented by its derivative depends only on the present value of $\phi$ and the present value of the noise. Hence $\phi(t)$ is a continuous Markov process, permitting us to use random walk techniques to determine its probability distribution. A mechanical analog is useful in understanding the mechanism of this "random walk." Consider the pendulum of Fig. 2 consisting of a weightless ball attached by an infinitesimally thin, weightless rod to a fixed point, and let the apparatus be horizontal on a table top which is being randomly agitated. The pendulum is free to turn a full revolution about the point. Let the rod be initially at an angle $\phi$ with respect to the vertical axis. Let an external force (such as a constant wind) be exerted on the ball in the vertical direction. Let the surface of the table be rough so that it produces a frictional force opposing motion of magnitude $f \dot{\phi}$. In addition, let the ball be equipped with an internal engine which exerts a constant force $F$ along the axis of motion. The random agitation of the table produces a force on the ball which may be represented by the two stationary, white, Gaussian processes of zero means $n_{1}(t)$ in the vertical direction, and $n_{2}(t)$ in the horizontal direction. Then by equating forces along the instantaneous axis of motion, we obtain:

$$
f \dot{\phi}+G \sin \phi=F-n_{1}(t) \sin \phi-n_{2}(t) \cos \phi
$$


If we divide by $f$ and identify $F / f$ with $\left(\omega-\omega_{0}\right), G / f$ with $A K$, and $1 / f$ with $K$, we see that Eq. (6) is the same as Eq. (5).

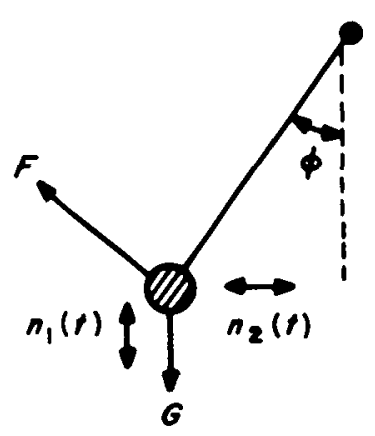

Fig. 2. Mechanical analog of the first-order loop

It is clear that in the absence of the random forces, the pendulum approaches the equilibrium position

$$
\phi_{0}=\sin ^{-1}(F / G)=\sin ^{-1}\left(\omega-\omega_{0}\right) /(A K)
$$

at which point the velocity is zero. Because this is a first-order system, there can be no overshoot. If $F>G$ or $\left(\omega-\omega_{0}\right)>A K$, there can be no equilibrium position, and the pendulum continues to revolve indefinitely which corresponds to a loop which can not achieve lock. When the random or noise forces are applied as well as the constant ones, the motion becomes a random walk, but when the noise variance is small, there is a strong tendency for the angle $\phi$ to approach and remain about this equilibrium position.

The complete statistical description of the random walk of the angle $\phi$ is given by its probability density as a function of time, $p(\phi, t)$. To understand qualitatively the behavior of this function, let us assume that the constant force $F=0$ and that initially (at $t=0$ ) the pendulum is at rest in the vertical position. Thus, $p(\phi, 0)=\delta(\phi)$. With the passage of time, the effect of the random forces will be felt in the movement of the pendulum from the equilibrium position. The qualitative behavior of the probability density function is sketched in Fig. 3. Of course, the condition

$$
\int_{-\infty}^{\infty} p(\phi, t) d \phi=1
$$

must always be met. After a sufficient amount of time, the random forces will push the pendulum around by more than half a revolution so that it will tend to return to the equilibrium position after a full cycle of rotation in either direction. This corresponds to the reference signal of the phase-locked loop advancing or retreating one cycle relative to the received signal. The average time for this occurrence depends on the signal-to-noise ratio. Thus after a sufficiently long period, the probability density will appear as a multimodal function, each mode being centered about equilibrium positions spaced $2 \pi$ rad apart, the central mode being the largest with each successive maximum progressively smaller. After an even longer period equal to several times the average time between revolutions, the central mode of the probability density will have diminished, the modes to either side will have become almost as large, and more modes of significant magnitude will have appeared. The central mode will remain the largest since the pendulum may have revolved in either direction with equal probability. Finally, in the steady state an arbitrary number of revolutions will have occurred. Then the probability density will be a periodic function (as will be proved in Part III). However, because the integral of the function must equal one at all times, the magnitude must be everywhere zero.

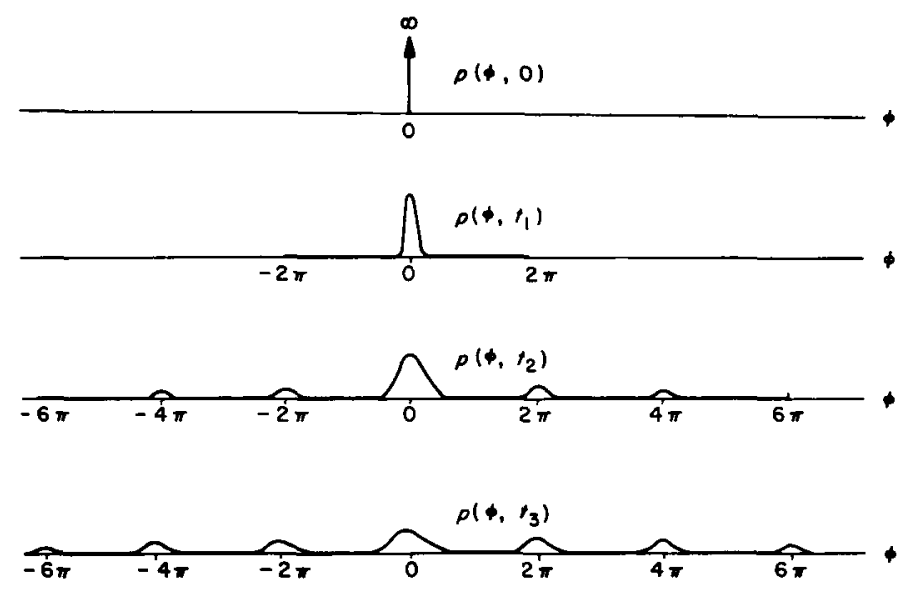

Fig. 3. Qualitative behavior of the probability density function for the first-order loop, $\omega=\omega_{0}$

In the case for which $F$ is not zero, or equivalently $\omega \neq \omega_{0}$, then clearly the pendulum will have a greater tendency to swing around in the sense corresponding to the direction of the force. Hence, the density function $p(\phi, t)$ will not be symmetrical. In either case, we are lead to realize that the significant parameter, at least in the steady state, is the angle (or phase error) $\phi$ modulo $2 \pi$, since the number of revolutions of the pendulum which have occurred does not affect the present state of the system. In fact, although $p(\phi, t)$ yields a complete 
description of the statistical behavior, it would appear that a combination of the steady-state distribution of $\phi$ modulo $2 \pi$ and the frequency or average time between revolutions would yield a simpler and equally valid representation. In the following Parts of this Report, these parameters will be obtained quantitatively.

\section{THE STEADY-STATE PHASE-ERROR PROBABILITY DENSITY FOR THE FIRST-ORDER LOOP}

A continuous random walk which is a Markov process is described by the statistical parameters of the incremental change of position as a function of the present position. Thus from Eq. (5), in the infinitesimal increment of time $\Delta t$, the phase will change by an amount ${ }^{1}$

$$
\begin{aligned}
\Delta \dot{\phi}= & \int_{t}^{t+\Delta t} \phi(t) d t=\left(\omega-\omega_{0}\right) \Delta t-(A K \sin \phi) \Delta t \\
& -K\left[\sin \phi \int_{t}^{t+\Delta t} n_{1}(u) d u+\cos \phi \int_{t}^{t+\Delta t} n_{2}(u) d u\right]
\end{aligned}
$$

Thus, since $n_{1}(t)$ and $n_{2}(t)$ are white, Gaussian processes with

$$
\overline{n_{1}(u)}=\overline{n_{2}(u)}=0
$$

and

$$
\overline{n_{1}(u) n_{1}(v)}=\overline{n_{2}(u) n_{2}(v)}=\left(N_{0} / 2\right) \delta(u-v)
$$

${ }^{1}$ This assumes that $\phi(t)$ is a continuous process, which is justified by physical considerations. it follows that for a given position $\phi, \Delta \phi$ is a Gaussian variable with mean

$$
\overline{\Delta \phi}=\left[\left(\omega-\omega_{0}\right)-A K \sin \phi\right] \Delta t
$$

and variance

$$
\begin{aligned}
\sigma_{\Delta \phi}^{2}=(\overline{\Delta \phi})^{2}-(\overline{\Delta \phi})^{2} \\
=K^{2}\left[\sin ^{2} \phi \int_{t}^{t+\Delta t} \int_{t}^{t+\Delta t} \overline{n_{1}(u) n_{1}(v)} d u d v\right. \\
\left.\quad \quad+\cos ^{2} \phi \int_{t}^{t+\Delta t} \int_{t}^{t+\Delta t} \overline{n_{2}(u) n_{2}(v)} d u d v\right] \\
=K^{2}\left(N_{0} / 2\right) \Delta t
\end{aligned}
$$

It is worth noting in passing that for the determination of $p(\phi ; t)$, Eq. (9) shows that the two noise terms could be replaced by a single noise $n^{\prime}(t)$ of the same spectral density so that Eq. (5) could be rewritten

$$
\dot{\phi}(t)=\left(\omega-\omega_{0}\right)-A K \sin \phi(t)-K n^{\prime}(t)
$$

which is conveniently represented by the block diagram of Fig. 4. The model can be shown trivially also to hold 
for a higher order loop in which the filter is included after the amplifier. The VCO of Fig. 1 is replaced by an integrator and the multiplier by an adder and sinusoidal nonlinearity. This differs from the linearized model of Ref. 4 only in the inclusion of the sinusoidal nonlinearity.

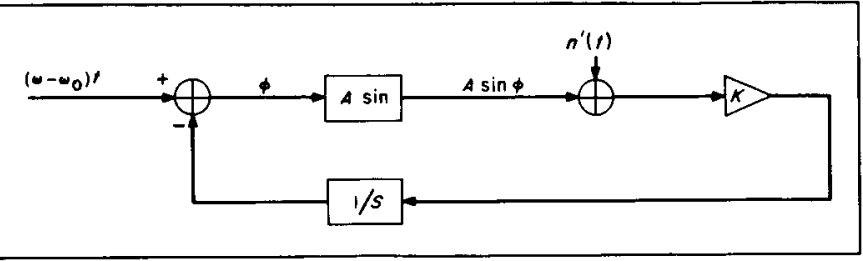

Fig. 4. Model of first-order loop

With the knowledge of the statistical parameters of the increment $\Delta \phi$, we may proceed to obtain $p(\phi, t)$. It was shown by Uhlenbeck and Ornstein (Ref. 11) and Wang and Uhlenbeck (Ref. 12) that for a continuous Markov process, the instantaneous probability density $p(\phi, t)$ must satisfy the partial differential equation

$$
\frac{\partial p(\phi, t)}{\partial t}=-\frac{\partial}{\partial \phi}[A(\phi) p(\phi, t)]+\frac{1}{2} \frac{\partial^{2}}{\partial \phi^{2}}[B(\phi) p(\phi, t)]
$$

with the initial condition

$$
p(\phi, 0)=\delta\left(\phi-\phi_{0}\right)
$$

where $\phi_{0}$ is the initial value of $\phi$, and where $A(\phi)$ and $B(\phi)$ are normalized moments of the infinitesimal increment given by the following expressions:

$$
\begin{aligned}
& A(\phi)=\lim _{\Delta t \rightarrow 0}(1 / \Delta t) \overline{\Delta \phi} \\
& B(\phi)=\lim _{\Delta t \rightarrow 0}(1 / \Delta t) \sigma_{\Delta \phi}^{2}
\end{aligned}
$$

Equation (11) is known as the Fokker-Planck equation or the diffusion equation because it is essentially the same as the equation for heat diffusion. From Eq. (8) and (9) we obtain

$$
\begin{aligned}
& A(\phi)=\left(\omega-\omega_{0}\right)-A K \sin \phi \\
& B(\phi)=K^{2} N_{0} / 2
\end{aligned}
$$

so that Eq. (11) becomes

$$
\frac{\partial p}{\partial t}=\frac{\partial}{\partial \phi}\left[\left(A K \sin \phi+\omega_{0}-\omega\right) p\right]+\frac{1}{4} K^{2} N_{0} \frac{\partial^{2} p}{\partial \phi^{2}}
$$

Although the equation is linear in $p$, the complete solution for $p(\phi, t)$ is somewhat complicated by the nonlinear behavior of the variable coefficients.

However, the result of greatest interest is the steadystate distribution

$$
p(\phi)=\lim _{t \rightarrow \infty} p(\phi, t)
$$

By definition, the steady-state distribution does not vary with time. Therefore,

$$
\frac{\partial p(\phi)}{\partial t}=\lim _{t \rightarrow \infty} \frac{\partial p(\phi, t)}{\partial t}=0
$$

Thus in the steady state, the partial differential Eq. (12) reduces to an ordinary differential equation in $p(\phi)$. Letting

$$
\alpha=(4 A) /\left(K N_{0}\right)
$$

and

$$
\beta=\left[4\left(\omega-\omega_{0}\right)\right] /\left(K^{2} N_{0}\right)
$$

we obtain from Eq. (12)

$$
0=\frac{d}{d \phi}\left[(\alpha \sin \phi-\beta) p(\phi)+\frac{d p(\phi)}{d \phi}\right]
$$

If we integrate once with respect to $\phi$, we obtain a first-order linear differential equation which is readily solved as ${ }^{2}$

$$
\begin{aligned}
& p(\phi)= C \exp (\alpha \cos \phi+\beta \phi) \\
& \times\left[1+D \int_{-\pi}^{\phi} \exp -(\alpha \cos x+\beta x) d x\right] \\
& \quad-\pi \leq \phi \leq \pi
\end{aligned}
$$

To evaluate the constants, we must utilize boundary conditions. First of all, as was pointed out in Part II, in the steady state we are interested in the distribution of $\phi$ modulo $2 \pi$. Thus, one condition is:

$$
p(\pi)=p(-\pi)
$$

Secondly, since $\phi$ must lie between $-\pi$ and $\pi$ and $p(\phi)$ is a probability density, it follows that

\footnotetext{
'The results of Eq. (17) through (21) were first obtained by V. I. Tikhonov (Ref. 9). Actually, these are a special case of an expression derived by Andronov, Pontryagin and Witt (Ref. 13) for a random walk problem with arbitrary nonlinear restoring forces.
} 


$$
\int_{-\pi}^{\pi} p(\phi) d \phi=1
$$

Using Eq. (18), we obtain

$$
D=\frac{\exp (-2 \beta \pi)-1}{\int_{-\pi}^{\pi} \exp -(\alpha \cos x+\beta x) d x}
$$

Then by means of Eq. (19), the constant $C$ can be evaluated.

In the special case $\beta=0$ (which requires $\omega=\omega_{0}$; i.e., when the VCO quiescent frequency is exactly at the frequency of the incoming signal), from Eq. (20) we see that $D=0$ and that when $\omega=\omega_{0}$, the probability density becomes

$$
p(\phi)=\frac{\exp (\alpha \cos \phi)}{2 \pi I_{0}(\alpha)} \quad-\pi \leq \phi \leq \pi
$$

since

$$
C=\frac{1}{\int_{-\pi}^{\pi} \exp (\alpha \cos \phi) d \phi}=\frac{1}{2 \pi I_{0}(\alpha)}
$$

The parameter a plays a very important role. From Eq. (15) we have

$$
\alpha=\frac{(4 A)}{\left(K N_{0}\right)}=\frac{\left(A^{2}\right)}{\left[N_{0}(A K / 4)\right]}
$$

But $A^{2}$ is the received signal power, while $A K / 4$ is an important parameter defined for the linearized model of the loop (Ref. 4). If we replace the sinusoidal nonlinearity in the model of Fig. 4 by its gain $A$ about $\phi=0$, we obtain the linearized model. Then the variance of $\phi$ is obtained by using Parseval's theorem as:

$$
\sigma_{\phi}^{2}=\frac{1}{2 \pi} \int_{-\infty}^{\infty} \frac{N_{0}}{2} \frac{K^{2} / \omega^{2}}{1+\left(A^{2} K^{2} / \omega^{2}\right)} d_{\omega}=N_{0}(A K / 4)
$$

The variance of $\phi$ is the same as the noise power at the output of an ideal low-pass filter of bandwidth $A K / 4$ when the input is white noise of one-sided spectral density $N_{0}$. Hence, for the first-order filter, the loop bandwidth is defined as

$$
B_{L}=A K / 4
$$

so that Eq. (22) becomes

$$
\alpha=\left(A^{2}\right) /\left(N_{0} B_{L}\right)
$$

which is the signal-to-noise ratio in the bandwidth of the loop.

Equation (21) is plotted in Fig. 5 for several values of $\alpha$. It resembles a Gaussian distribution for large signalto-noise ratios, $\alpha$, and becomes flat as $\alpha$ approaches zero. The asymptotic behavior of Eq. (21) for large $\alpha$ is of interest. Since for large $\alpha$

$$
\begin{gathered}
I_{0}(\alpha) \sim(\exp \alpha) /(2 \pi \alpha)^{3 / 2} \\
p(\phi)=\frac{[\exp (\alpha \cos \phi)]}{\left[2 \pi I_{0}(\alpha)\right]} \sim \frac{\{\exp [\alpha(\cos \phi-1)]\}}{(2 \pi / \alpha)^{3 / 2}}
\end{gathered}
$$

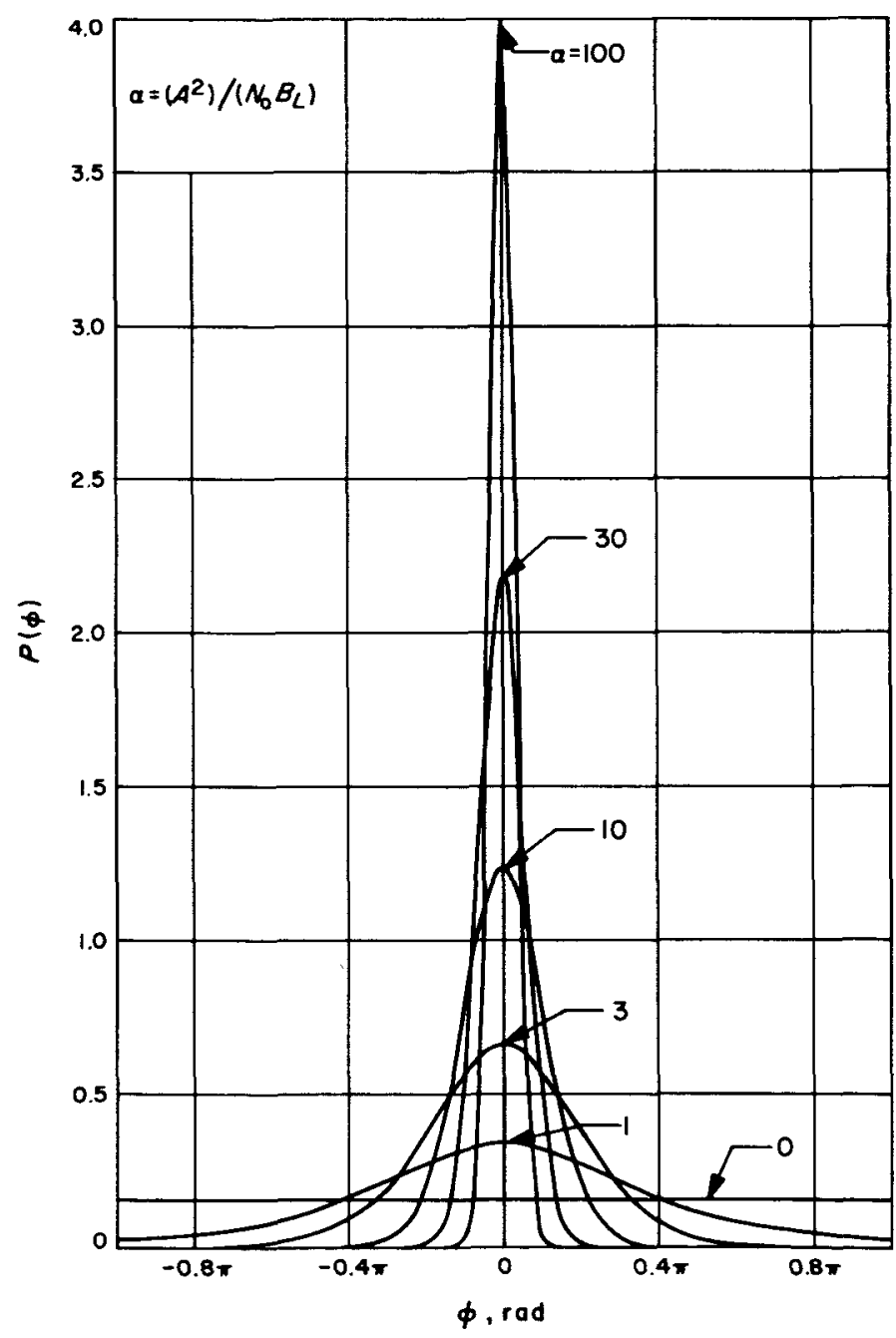

Fig. 5. First-order loop, steady-state probability densities for $\omega=\omega_{0}$ 
Expanding $\cos \phi$ in a Taylor series, we obtain

$$
p(\phi) \sim \frac{\exp \left[\frac{-\alpha \phi^{2}}{2}\left(1-\frac{2 \phi^{4}}{4 !}+\frac{2 \phi^{6}}{6 !} \cdots\right)\right]}{(2 \pi / \alpha)^{1 / 2}}-\pi \leq \phi \leq \pi
$$

When $\alpha$ is large, $p(\phi)$ decays rapidly so that the function is very small for all but very small values of $\phi$. Thus the higher order terms of the series representation of $\cos \phi$ have very little effect for moderate values of $p(\phi)$. Hence the graph of $p(\phi)$ will appear to be nearly Gaussian for large $\alpha$, and in this case, the results of the linear model are quite accurate.

The cumulative steady-state probability distribution

$$
P\left(|\phi|<\phi_{0}\right)=\int_{-\phi_{0}}^{\phi_{0}} p(\phi) d \phi \quad 0<\phi_{0}<\pi
$$

is also of interest since it indicates the percentage of time during which the absolute value of the loop phase error $\phi$ is less than a given magnitude $\phi_{0}$. This may be calculated when $\omega=\omega_{0}$ in the following manner. Expanding $p(\phi)$ of Eq. (21) in a Fourier series, we have

$$
\begin{aligned}
p(\phi) & =\frac{\exp (\alpha \cos \phi)}{2 \pi I_{0}(\alpha)} \\
& =\frac{1}{2 \pi I_{0}(\alpha)}\left[I_{0}(\alpha)+2 \sum_{n=1}^{\infty} I_{n}(\alpha) \cos n \phi\right]
\end{aligned}
$$

Then

$$
\begin{aligned}
& P\left(|\phi|<\phi_{0}\right)= 2 \int_{0}^{\phi_{0}} p(\phi) d(\phi) \\
&= \frac{\phi_{0}}{\pi}+\frac{2}{\pi} \sum_{n=1}^{\infty} \frac{I_{n}(\alpha) \sin n \phi_{0}}{n I_{0}(\alpha)} \\
& 0<\phi_{0}<\pi \text { for } \omega=\omega_{0}
\end{aligned}
$$

This series converges rapidly so that Eq. (26) could be calculated for several values of $\alpha$ without the use of a large-scale digital computer. The results are shown in Fig. 6.
The variance of $\phi$ can be similarly obtained.

$$
\begin{aligned}
\sigma_{\phi}^{2} & =\int_{-\pi}^{\pi} \phi^{2} \exp (\alpha \cos \phi) d \phi \\
& =\frac{1}{2 \pi I_{0}(\alpha)} \int_{-\pi}^{\pi} \phi^{2}\left[I_{0}(\alpha)+2 \sum_{n=1}^{\infty} I_{n}(\alpha) \cos n \phi\right] d \phi \\
& =\frac{\pi^{2}}{3}+4 \sum_{n=1}^{\infty} \frac{(-1)^{n} I_{n}(\alpha)}{n^{2} I_{0}(\alpha)}
\end{aligned}
$$

This series converges even more rapidly than that of Eq. (26). It was computed manually and is plotted in Fig. 7 as a function of $1 / \alpha$. Note that as the SNR $\alpha$ approaches zero, the variance approaches $\pi^{2} / 3$ which is the variance of a random variable that is uniformly distributed from $-\pi$ to $+\pi$.

For the general case $\left(\omega \neq \omega_{0}\right)$, Eq. (17), (19), and (20) yield the entire distribution. However, analog or digital computation is required to evaluate the pertinent integrals. The case for which $(\beta / \alpha)=\left(\omega-\omega_{0}\right) /(A K)=\sin (\pi / 4)$ is shown in Fig. 8. The constants as well as the distribution were obtained by means of the analog computer.

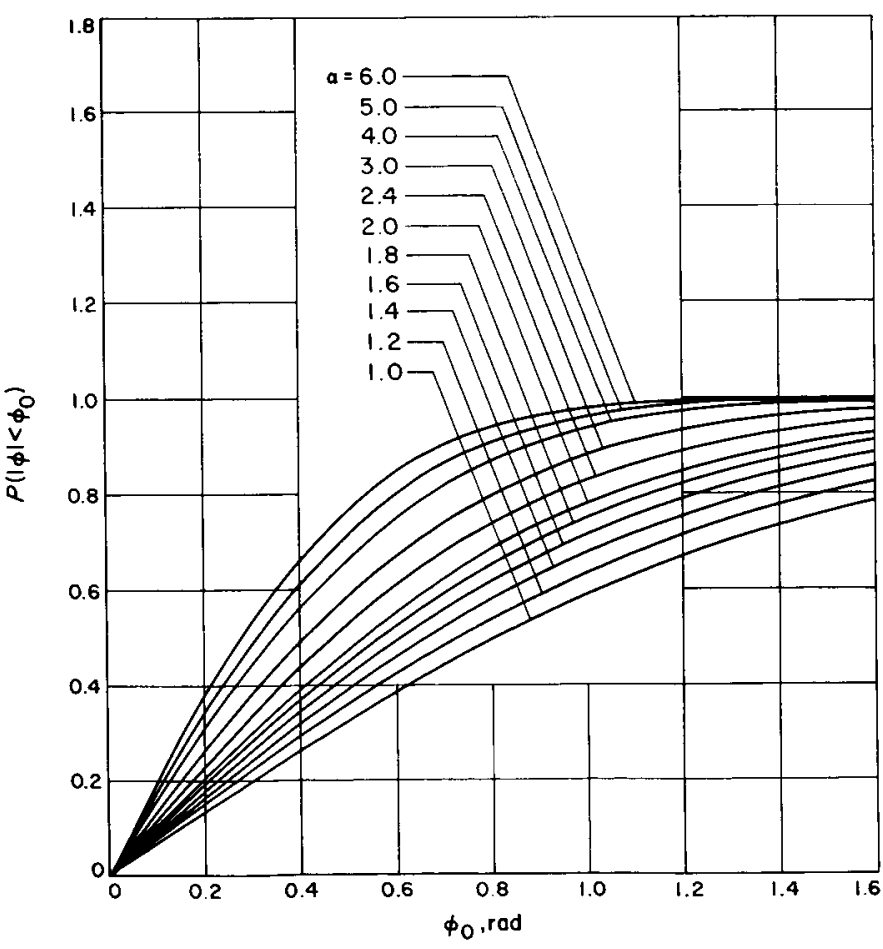

Fig. 6. Steady-state, cumulative probability distribution of first-order loop for $\omega=\omega_{0}$ 


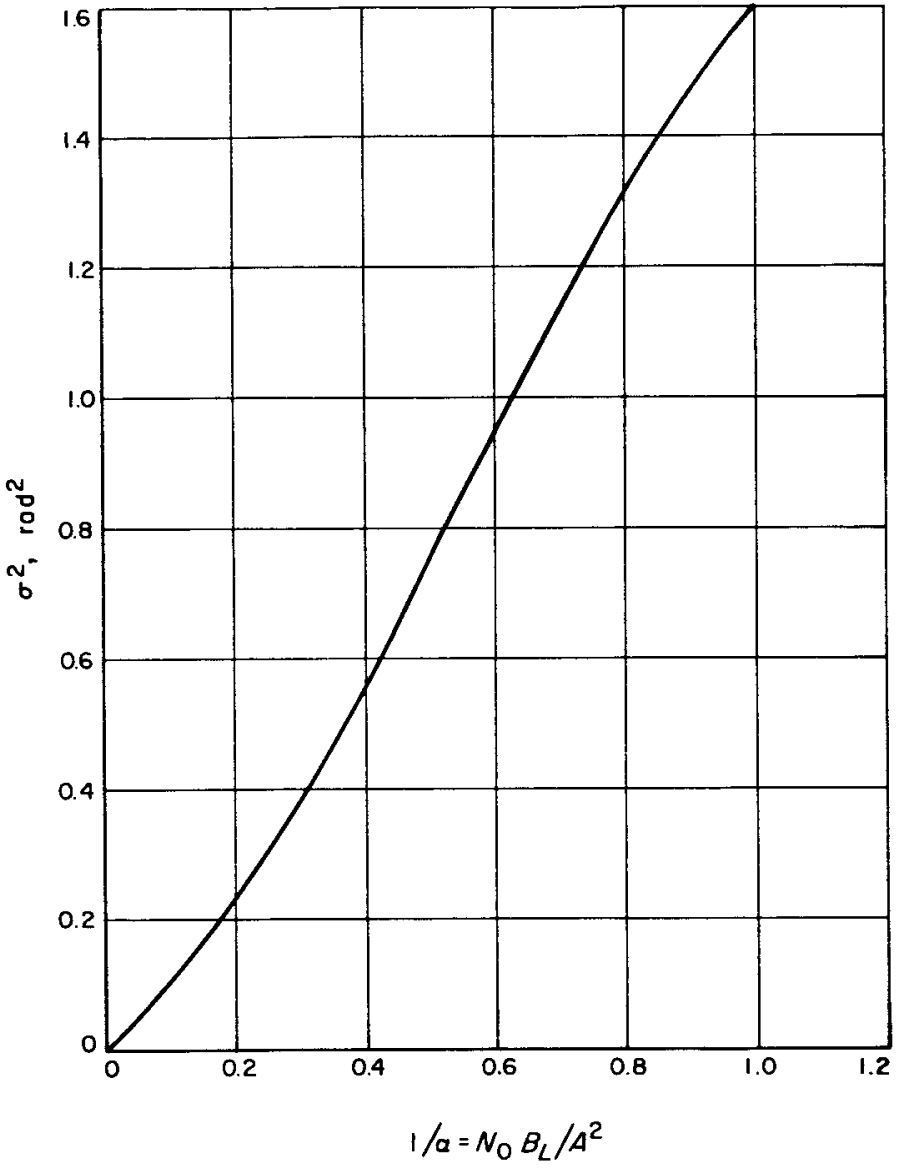

Fig. 7. Variance of phase-error for first-order loop where $\omega=\omega_{0}$

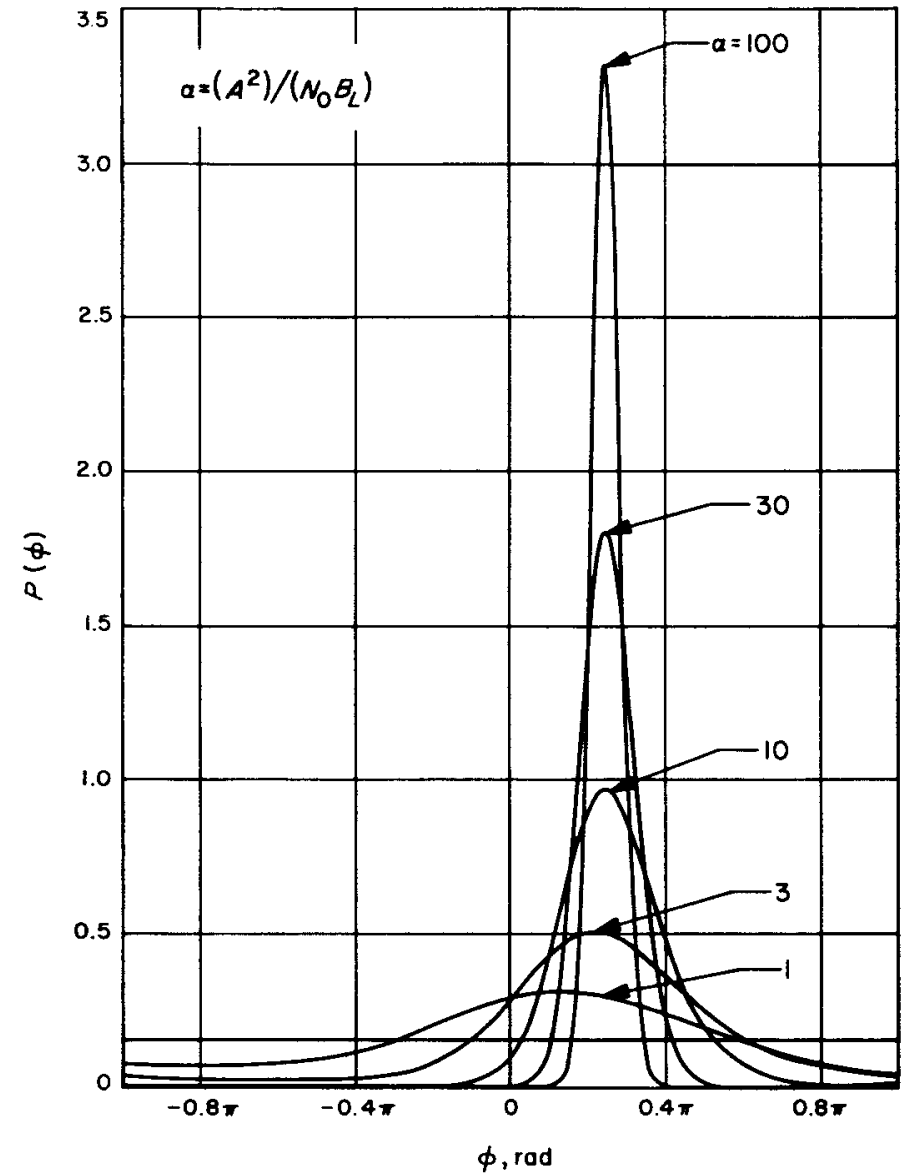

Fig. 8. First-order loop, steady-state probability densities for $\left(\omega=\omega_{0}\right) /(A K)=\sin (\pi / 4)$ 


\section{THE FOKKER-PLANCK EQUATION FOR HIGHER-ORDER LOOPS}

Consider the phase-locked loop whose filter has the rational transfer function

$$
F(s)=G(s) / H(s)
$$

where $G(s)$ and $H(s)$ are polynomials such that $G(0)=1$, $H(0)=0$

$$
\operatorname{deg} G(s) \leq \operatorname{deg} H(s)=n-1
$$

then

$$
\begin{array}{ll}
G(s)=\sum_{k=0}^{n-1} a_{k} s^{k} & a_{0} \neq 0 \\
H(s)=\sum_{k=1}^{n-1} b_{k} s^{k} & b_{n-1} \neq 0
\end{array}
$$

This will be referred to as an $n$ th-order loop. In this case, Eq. (4) which describes the operation of the loop becomes

$$
s H(s) \phi=-K G(s)\left[\left(A+n_{1}\right) \sin \phi+n_{2} \cos \phi\right]
$$

since

$$
s^{k}\left(\omega-\omega_{0}\right)=\frac{d k}{d t}\left(\omega-\omega_{0}\right)=0 \quad \text { for } k \geqslant 1
$$

The reason for the pole at the origin of $F(s)$ is now clear. It eliminates the constant $\left(\omega-\omega_{0}\right)$ which causes the steady-state phase error in the first-order loop. Now let us define the random variable $\varepsilon$ by the relation ${ }^{3}$

$$
\phi=G(s) \varepsilon
$$

Inserting this in Eq. (29), we obtain

$$
s H(s)_{\varepsilon}=-K\left\{\left[A+n_{1}\right] \sin [G(s) \varepsilon]+n_{2} \cos [G(s) \varepsilon]\right\}
$$

which is an $n$ th-order differential equation. Now let us define the $n$ random variables $x_{0}, x_{1}, \cdots, x_{n-1}$ as

$$
\boldsymbol{x}_{k}=\frac{d^{k} \varepsilon}{d t^{k}} \quad k=0,1, \cdots, n-1
$$

Inserting these for the derivatives of $\varepsilon$ in Eq. (31) and by using Eq. (28), we obtain

'This substitution which leads to the representation of $\phi$ as the sum of the components of a Markov vector (Eq. 33) was suggested by J. N. Franklin.

$$
\begin{aligned}
b_{n-1} \dot{x}_{n-1}+\sum_{k=1}^{n-2} b_{k} x_{k+1}= & -K\left[\left(A+n_{1}\right) \sin \left(\sum_{k=0}^{n-1} a_{k} x_{k}\right)\right. \\
& \left.+n_{2} \cos \left(\sum_{k=0}^{n-1} a_{k} x_{k}\right)\right]
\end{aligned}
$$

Also, we have

$$
x_{k}=\frac{d}{d t} \frac{d^{k-1} \varepsilon}{d t^{k-1}}=\dot{x}_{k-1}
$$

so that we may express the derivatives $\dot{x}_{k}$ in terms of the variables $x_{k}$ by the $n$ differential equations

$$
\begin{aligned}
& \dot{x}_{n-2}=x_{n-1} \\
& \dot{:} \quad \\
& \dot{x_{0}}=
\end{aligned}
$$$$
\dot{x}_{n-1}=-\sum_{k=1}^{n-2} \frac{b_{k}}{b_{n-1}} x_{k+1}-\frac{K}{b_{n-1}}
$$$$
\times\left[\left(A+n_{1}\right) \sin \left(\sum_{k=0}^{n-1} a_{k} x_{k}\right)+n_{z} \cos \left(\sum_{k=0}^{n-1} a_{k} x_{k}\right)\right]
$$

It follows also from Eq. (28), (30), and (32) that

$$
\phi=\sum_{k=0}^{n-1} a_{k} s^{k}
$$

The random vector $\left(x_{0}, \cdots, x_{n-1}\right)$ is a Markov vector since an incremental change depends only on the present state of the vector.

Wang and Uhlenbeck (Ref. 12) have shown that for a vector Markov process $\mathrm{x}=\left(x_{0}, x_{1}, \cdots, x_{\mathrm{n}-1}\right)$, the Fokker-Planck equation is

$$
\begin{aligned}
\frac{\partial P(x)}{\partial t}= & -\sum_{k=0}^{n-1} \frac{\partial}{\partial x_{k}}\left[A_{k}(\mathbf{x}) P(\mathbf{x})\right] \\
& +\frac{1}{2} \sum_{k=0}^{n-1} \sum_{l=0}^{n-1} \frac{\partial^{2}}{\partial x_{k} \partial x_{l}}\left[B_{k l}(\mathbf{x}) P(\mathbf{x})\right]
\end{aligned}
$$

where

$$
A_{k}(\mathbf{x})=\lim _{\Delta t \rightarrow 0} \frac{1}{\Delta t}\left(\overline{\Delta x}_{k}\right)
$$

and

$$
B_{k l}(\mathbf{x})=\lim _{\Delta t \rightarrow 0} \frac{1}{\Delta t}\left(\overline{\left.\Delta x_{k}\right)\left(\Delta x_{l}\right.}\right)
$$


with the initial condition

$$
\begin{gathered}
P(\mathbf{x}, 0)=\prod_{k=0}^{n-1} \delta\left(x_{k}-x_{k, 0}\right) \\
A_{k}(\mathbf{x})=x_{k+1} \text { for } k=0,1, \cdots, n-2 \\
A_{n-1}(\mathbf{x})=-\sum_{k=1}^{n-2} \frac{b_{k}}{b_{n-1}} x_{k+1}-\frac{K A}{b_{n-1}} \sin \sum_{k=0}^{n-1} a_{k} x_{k} \\
B_{n-1, n-1}(x)=\lim _{\Delta t \rightarrow 0} \frac{1}{\Delta t} \frac{K^{2}}{b_{n-1}^{2}} \\
\times\left[\sin ^{2} \phi \int_{t}^{t+\Delta t} \int_{t}^{t+\Delta t} \overline{n_{1}(u) n_{1}(v)} d u d v\right. \\
\left.+\cos ^{2} \phi \int_{t}^{t+\Delta t} \int_{t}^{t+\Delta t} \overline{n_{2}(u) n_{2}(v)} d u d v\right] \\
=\frac{K^{2} N_{0} / 2}{b_{n-1}^{2}} \\
B_{k, l}(\mathbf{x})=0 \text { for all } k \neq n-1 \text { and } l \neq n-1
\end{gathered}
$$

Thus, the Fokker-Planck equation for the nth-order loop is

$$
\begin{aligned}
\frac{\partial P(x, t)}{\partial t}= & -\sum_{k=0}^{n-2} x_{k+1} \frac{\partial P(x, t)}{\partial x_{k}}+\frac{1}{b_{n-1}} \frac{\partial}{\partial x_{n-1}} \\
& \times\left[\left(\sum_{k=0}^{n-2} b_{k} x_{k+1}+K A \sin \sum_{k=0}^{n-1} a_{k} x_{k}\right) P(x, t)\right] \\
& +\frac{K^{2} N_{0}}{4 b_{n-1}^{2}} \frac{\partial^{2} P(x, t)}{\partial x_{n-1}^{2}}
\end{aligned}
$$

where

$$
\phi=\sum_{k=0}^{n-1} a_{k} x_{k}
$$

Solution of this general case does not appear possible. However, in the next Section some results are obtained for the second-order loop. 


\section{STEADY-STATE PROBABILITY DISTRIBUTION FOR THE SECOND-ORDER LOOP}

The loop filter of greatest interest ${ }^{4}$ is

$$
F(s)=1+(a / s)=(s+a) /(s)
$$

which requires a single integrator with gain $a$. In terms of the parameters of Eq. (28), $n=2, a_{0}=a, a_{1}=1$, $b_{1}=1$. Substituting these parameters in Eq. (33) and (36), we obtain the differential equations for the random variables

$$
\begin{aligned}
& \dot{x}_{1}=-K\left[\left(A+n_{1}\right) \sin \left(a x_{0}+x_{1}\right)\right]-K n_{2} \cos \left(a x_{0}+x_{1}\right) \\
& \dot{x}_{0}=x_{1}
\end{aligned}
$$

and the Fokker-Planck equation

$$
\frac{\partial P}{\partial t}=-x_{1} \frac{\partial p}{\partial x_{0}}+\frac{\partial}{\partial x_{1}}\left[\left(A K \sin \left(a x_{0}+x_{1}\right) P\right]+\frac{K^{2} N_{6}}{4} \frac{\partial^{2} P}{\partial x_{1}^{2}}\right.
$$

where

$$
\phi=a x_{0}+x_{1}
$$

If we restrict our attention to the steady-state probability distribution

$$
P\left(x_{0}, x_{1}\right)=\lim _{t \rightarrow \infty} P\left(x_{0}, x_{1}, t\right)
$$

since

$$
\lim _{t \rightarrow \infty} \frac{d P}{d t}\left(x_{0}, x_{1}, t\right)=0
$$

we obtain

$$
x_{1} \frac{\partial P}{\partial x_{0}}=A K \frac{\partial}{\partial x_{1}}\left[\sin \left(a x_{0}+x_{1}\right) P\right]+\frac{K^{2} N_{0}}{4} \frac{\partial^{2} P}{\partial x_{1}^{2}}
$$

With the substitutions

$$
\begin{aligned}
& \phi=a x_{0}+x_{1} \\
& z=a x_{0}
\end{aligned}
$$

we obtain an equation in $P(\phi, z)$ (note that the Jacobian of the transformation is $a$ )

'Tikhonov (Ref. 10) considered the RC low-pass filter whose transfer function is $1 /(s+b)$. Its value is questionable, however, since it does not reduce the mean phase error to zero, as the perfect integrator does.

$$
a(\phi-z)\left(\frac{\partial P}{\partial \phi}+\frac{\partial P}{\partial z}\right)=A K \frac{\partial}{\partial \phi}(\sin \phi P)+\frac{K^{2} N_{0}}{4} \frac{\partial^{2} P}{\partial \phi^{2}}
$$

Even this partial differential equation cannot be solved directly. However, since we are interested only in the distribution of $\phi$

$$
p(\phi)=\int_{-\infty}^{\infty} P(\phi, z) d z
$$

we may integrate both sides of Eq. (41) with respect to $z$ over the infinite line and obtain an ordinary differential equation in $p(\phi)$

$$
\begin{aligned}
& a\left\{\frac{d(\phi p)}{d \phi}-\frac{d}{d \phi}\left[\int_{-\infty}^{\infty} z P(\phi, z) d z\right]\right\}=A K \frac{d}{d \phi}(\sin \phi p) \\
& \quad+\frac{K^{2} N_{0}}{4} \frac{d^{2} p}{d \phi^{2}}
\end{aligned}
$$

But

$$
\int_{-\infty}^{\infty} z P(\phi, z) d z=p(\phi) \int_{-\infty}^{\infty} z P(z \mid \phi) d z=p(\phi) E(z \mid \phi)
$$

so that Eq. (42) becomes

$$
0=\frac{d}{d \phi}\left\{\left[A K \sin \phi-a_{\phi}+a E(z \mid \phi)\right] p+\frac{K^{2} N_{0}}{4} \frac{d p}{d \phi}\right\}
$$

Unfortunately, it is not possible to determine exactly $E(z \mid \phi)$, which is a function of $\phi$, without knowing $P(z, \phi)$, which would require solution of Eq. (41). However, its general form can be obtained as follows: from Eq. (40) we have $z=\phi-x_{1}$ so that

$$
\begin{aligned}
E[z(t) \mid \phi(t)] & =E\left[\phi(t)-x_{1}(t) \mid \phi(t)\right] \\
& =\phi(t)-E\left[x_{1}(t) \mid \phi(t)\right]
\end{aligned}
$$

Integrating Eq. (37) using Eq. (40), we have

$$
\begin{aligned}
x_{1}(\infty) & -x_{1}(t)=-A K \int_{t}^{\infty} \sin \phi(\xi) d \xi \\
& -K \int_{t}^{\infty} n_{1}(\xi) \sin \phi(\xi) d \xi-K \int_{t}^{\infty} n_{2}(\xi) \cos \phi(\xi) d \xi
\end{aligned}
$$


Since the noise is white, $n_{1}(t)$ and $n_{2}(t)$ are independent of $\phi(t)$ for all $t$ so that, since $\overline{n_{1}(t)}=\overline{n_{2}(t)}=0$, the expectations of the noise terms are zero. Also

$$
E\left[x_{1}(\infty) \mid \phi(t)\right]=E\left[x_{1}(\infty)\right]=0
$$

since it is clear that the mean of the process is zero. Therefore,

$$
E\left[x_{1}(t) \mid \phi(t)\right]=A K \int_{t}^{\infty} E[\sin \phi(\xi) \mid \phi(t)] d \xi
$$

This is the integral of the expectation of $\sin \phi$ over the entire past history of the process given the present value of $\phi$. Combining Eq. (43), (44), and (45), and letting $\xi=t+\tau$, we obtain

$$
\begin{aligned}
0= & \frac{d}{d \phi}\left\{\frac{4 A}{K N_{0}}\left(\sin \phi-a \int_{0}^{\infty} E[\sin \phi(t+\tau) \mid \phi(t)] d \tau\right)\right. \\
& \left.\times p(\phi)+\frac{d p(\phi)}{d \phi}\right\}
\end{aligned}
$$

The magnitude of the expectation is always less than one and becomes negligible for values of $\tau$ several times the inverse bandwidth of the spectrum of $\phi(t)$. This bandwidth is proportional to $A K$, as we found for the first-order loop. Therefore, the order of magnitude of the integral is inversely proportional to $A K$, and if $a<<A K$, the second term in the coefficient of $p(\phi)$ is much smaller than the first. Neglecting this second term reduces Eq. 46 to the steady-state Fokker-Planck equation for the first-order loop (Eq. 16) with $\omega=\omega_{0}$, whose solution is Eq. (21). Thus when the second integrator gain $a<<A K$,

$$
p(\phi) \simeq \frac{\exp (\alpha \cos \phi)}{2 \pi I_{0}(\alpha)} \quad-\pi \leqslant \phi \leqslant \pi
$$

On the other hand, for any value of $a$ when the SNR is large enough, $\phi(t)$ will be small for all time so that $\sin \phi(t) \simeq \phi(t)$ and both $\phi(t)$ and $\sin \phi(t)$ will be nearly Gaussian processes. In this case, the expectation can be approximated by

$$
\int_{0}^{\infty} E[\sin \phi(t+\tau) \mid \phi(t)] d \tau \simeq\left[\int_{0}^{\infty} \rho_{\phi}(\tau) d \tau\right] \sin \phi
$$

where $\rho_{\phi}(\tau)$ is the normalized autocorrelation function of the stationary process $\phi(t)$. The integral can be obtained by using Parseval's theorem:

$$
\int_{0}^{\infty} \rho_{\phi}(\tau)=\frac{1}{2 \sigma^{2}} \int_{-\infty}^{\infty} R_{\phi}(\tau) d \tau=\frac{S_{\phi}(0)}{2 \sigma^{2}}
$$

where $R_{\phi}(\tau)$ is the unnormalized autocorrelation function, $\sigma^{2}$ the variance of $\phi$, and $S_{\phi}(\omega)$ the spectral density. Since we have approximated $\sin \phi$ by $\phi$, we may use the linearized version of Fig. 4 with the loop filter $F(s)=[1+(a / s)]$ inserted. Then

$$
S_{\phi}(\omega)=\frac{N_{0} K^{2}}{2}\left|\frac{s+a}{s^{2}+A K s+a A K}\right|^{2}
$$

so that $S_{\phi}(0)=\left(N_{0}\right) /\left(2 A^{2}\right)$.

$$
\sigma^{2}=\frac{1}{2 \pi} \int_{-\infty}^{\infty} S_{\phi}(\omega) d \omega=\frac{N_{0}}{4 A^{2}}(A K+a)
$$

and

$$
\int_{0}^{\infty} \rho_{\phi}(\tau) d \tau=1 /(A K+a)
$$

Inserting this integral in Eq. (48) and substituting in Eq. (46), we obtain

$$
0=\frac{d}{d \phi}\left\{\frac{4 A}{K N_{0}}\left[\sin \phi\left(\frac{A K}{A K+a}\right)\right] p(\phi)+\frac{d p(\phi)}{d \phi}\right\}
$$

whose solution with the boundary conditions of Eq. (18) and (19) is

$$
p(\phi) \simeq \frac{\exp \left(\alpha^{\prime} \cos \phi\right)}{2 \pi I_{0}\left(\alpha^{\prime}\right)} \quad \text { for large } \alpha^{\prime}
$$

where the effective $\mathrm{SNR}, \alpha^{\prime}$, is given by

$$
\alpha^{\prime}=\left(A^{2}\right) /\left[N_{0}(A K+a) /(4)\right]
$$

If we let $B_{L}=(A K+a) / 4$, this is the same expression as that for the first-order loop with $\omega=\omega_{0}$. As would be expected, this expression for loop bandwidth for the second-order loop is that obtained from the linear model of the loop. 


\section{MEAN TIME TO LOSS OF LOCK AND FREQUENCY OF SKIPPING CYCLES}

Since we have obtained only solutions for steady-state probabilities, a valuable statistic is the expected time required for the absolute value of the phase error to exceed some value $\phi_{l}$ when it is initially zero. When this occurs, the loop will be said to have lost lock. Of particular interest is the case for which $\phi_{l}= \pm 2 \pi$, which represents a loss or gain of a complete cycle, or for the mechanical analog, a complete revolution of the pendulum.

We only treat the case of the first-order loop for which the received frequency $\omega$ equals the VCO quiescent frequency $\omega_{0}$ so that $\phi=0$ is the equilibrium position. This is also a good approximation to the steady-state behavior of the second-order loop with any value of $\omega-\omega_{0}$ but with very small integrator gain $a$, as will be discussed later in this Part. For the first-order loop, when $\omega \neq \omega_{0}$, the same approach can be used measuring phase error from the equilibrium position rather than from zero, but the results are in the form of integrals which require numerical calculation.

Returning to the mechanical analog of the pendulum of Part II, we treat the motion of the ball by the operational equation

$$
\dot{\phi}=-A K \sin \phi-K n_{1} \sin \phi-K n_{2} \cos \phi
$$

as long as $|\phi|<\phi_{l}$. But when the pendulum angle $\phi$ reaches $\pm \phi_{l}$, we assume that it is grasped by a demon and removed from operation forever after. We seek the average time for this event to occur when the pendulum is initially at rest at $\phi=0$. As long as $|\phi|<\phi_{l}$, the probability density of $\phi$ is described in the same manner as before by the Fokker-Planck equation

$$
\begin{gathered}
\frac{\partial p}{\partial t}=\frac{\partial}{\partial \phi}(A K \sin \phi p)+\frac{N_{0} K^{2}}{4} \frac{\partial^{2} p}{\partial \phi^{2}} \\
p(\phi, 0)=\delta(\phi) \text { for }|\phi|<\phi_{l}
\end{gathered}
$$

However, as soon as $|\phi|$ reaches $\phi_{l}$ for the first time, the pendulum is removed from action so that

$$
p(\phi, t)=0 \text { for all }|\phi| \geq_{\phi_{l}}
$$

Thus we have the boundary conditions ${ }^{5}$

$$
p\left(\phi_{l}, t\right)=p\left(-\phi_{l}, t\right)=0
$$

Solution of Eq. (50) over the interval $-\phi_{l}<\phi<\phi_{l}$ with the boundary conditions of Eq. (51) would yield the probability density $p(\phi, t)$. Its integral over the interval

$$
\psi(t)=\int_{-\phi_{l}}^{\phi_{l}} p(\phi, t) d \phi
$$

gives the probability that $\phi$ has not yet reached $\phi_{l}$ at time $t$. Then the probability density of the time when $|\phi|$ reaches $\phi_{l}$ is $-[\partial \psi(t)] /(\partial t)$. Thus the expected time to reach the out-of-lock position $\phi_{l}$ is

$$
T=\int_{0}^{\infty}-t \frac{\partial \psi(t)}{\partial t}=-[t \psi(t)]_{0}^{\infty}+\int_{0}^{\infty} \psi(t) d t
$$

Since with probability $1,|\phi|$ must reach $\phi_{l}$ before $t=\infty$, then $\psi(\infty)=0$ so that the combination of Eq. (52) and (53) yields the mean time to lose lock

$$
T=\int_{0}^{\infty} \int_{-\phi_{l}}^{\phi_{l}} p(\phi, t) d \phi d t
$$

Now if we integrate both sides of Eq. (50) with respect to $t$ over the infinite interval, we obtain

$$
p(\phi, \infty)-p(\phi, 0)=\frac{\partial}{\partial \phi}(A K \sin \phi P)+\frac{N_{0} K^{2}}{4} \frac{\partial^{2} P(\phi)}{\partial \phi^{2}}
$$

where

$$
P(\phi)=\int_{0}^{\infty} p(\phi, t) d t
$$

As we noted previously, $p(\phi, \infty)=0$, and since $\phi$ is assumed initially at zero, $p(\phi, 0)=\delta(\phi)$. Therefore, we have

$$
-\delta(\phi)=\frac{\partial}{\partial \phi}[A K \sin \phi P(\phi)]+\frac{N_{0} K^{2}}{4} \frac{\partial^{2} P(\phi)}{\partial \phi^{2}}
$$

${ }^{5}$ The solution of the so-called first passage time problem by means of the Fokker-Planck equation with absorbing boundaries was first treated by Siegert (Ref. 14). 
which may be solved using the boundary conditions

$$
\begin{gathered}
P\left(\phi_{l}\right)=\int_{0}^{\infty} p\left(\phi_{l}, t\right) d t=0 \\
P\left(-\phi_{l}\right)=\int_{0}^{\infty} p\left(-\phi_{l}, t\right) d t=0
\end{gathered}
$$

The solution to Eq. (56) may then be integrated with respect to $\phi$ over the interval $-\phi_{l}, \phi_{l}$ to obtain $T$, the expected time to lose lock of Eq. (54). Taking the indefinite integral of both sides of Eq. (56), we obtain

$$
C-u(\phi)=A K \sin \phi P(\phi)+\frac{N_{0} K^{2}}{4} \frac{\partial P(\phi)}{\partial \phi}
$$

where $C$ is a constant to be evaluated from the boundary conditions. The solution to the first-order differential equation is

$$
\begin{aligned}
P(\phi)= & D \exp (\alpha \cos \phi) \\
& +\exp (\alpha \cos \phi) \int_{-\phi_{1}}^{\phi} \frac{\exp (-\alpha \cos x)}{\gamma}[C-u(x)] d x
\end{aligned}
$$

where

and

$$
\alpha=\frac{A^{2}}{N_{0}(A K / 4)}
$$

$$
\gamma=\frac{N_{0} K^{2}}{4}=\frac{A K}{\alpha}=\frac{4 B_{L}}{\alpha}
$$

Applying the boundary conditions of Eq. (57) yields the values of the constants as $D=0$ and $C=1 / 2$.

Thus

$$
P(\phi)=\frac{\exp (\alpha \cos \phi)}{\gamma} \int_{-\phi_{1}}^{\phi} \exp (-\alpha \cos x)\left[\frac{1}{2}-u(x)\right] d x
$$

and integrating with respect to $\phi$ over the interval $\left[-\phi_{l}, \phi_{l}\right]$, we obtain an expression for the mean time to lose lock

$$
\begin{aligned}
T & =\int_{\phi_{l}}^{\phi_{l}} P(\phi) d \phi=\frac{1}{\gamma} \int_{-\phi_{l}}^{\phi_{l}} d \phi \\
& \times \int_{\phi_{l}}^{\phi_{t}} \exp \alpha(\cos \phi-\cos x)\left[\frac{1}{2}-u(x)\right] d x \\
& =\frac{1}{\gamma} \int_{0}^{\phi_{l}} \int_{\phi}^{\phi_{l}} \exp \alpha(\cos \phi-\cos x) d x d \phi
\end{aligned}
$$

The domain of integration is the right isosceles triangle shown in Fig. 9. We can obtain a series representation of this double integral by expanding the integrands in Fourier series

$$
\exp (\alpha \cos \phi)=I_{0}(\alpha)+2 \sum_{m=1}^{\infty} I_{m}(\alpha) \cos m \phi
$$

$$
\exp (-\alpha \cos x)=I_{0}(\alpha)+2 \sum_{n=1}^{\infty}(-1)^{n} I_{n}(\alpha) \cos n x
$$

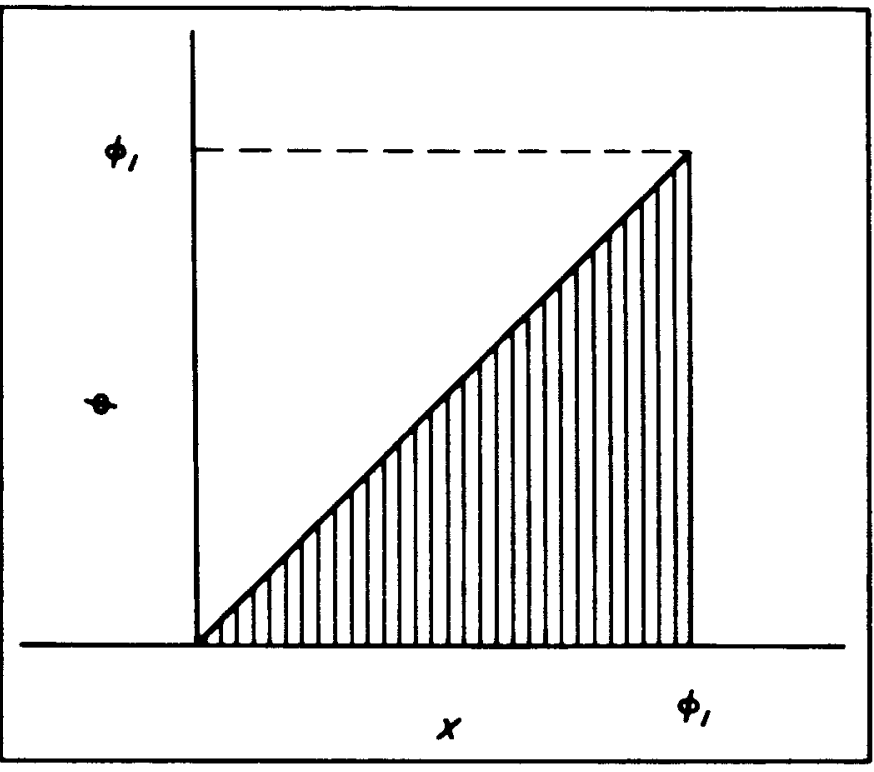

Fig. 9. Domain of integral $T$

Then

$$
\begin{aligned}
& T=\frac{1}{\gamma} \int_{0}^{\phi_{2}} \int_{\phi}^{\phi_{t}} \\
& \times\left[\begin{array}{cc}
I_{0}^{2}(\alpha)+4 I_{0}(\alpha) & \sum_{n=2,4,6, \cdots}^{\infty}(-1)^{n} I_{n}(\alpha) \cos n \phi \\
+4 \sum_{m=1}^{\infty} \sum_{n=1}^{\infty}-(1)^{n} I_{m}(\alpha) I_{n}(\alpha) \cos m \phi \cos n x
\end{array}\right] d \phi d x \\
& =\frac{1}{\gamma}\left[\frac{I_{0}^{2}(\alpha) \phi^{2}}{2}+4 I_{0}(\alpha) \sum_{n=1}^{\infty} \frac{I_{2 n}(\alpha)}{2 n} \sin 2 n \phi_{l}\right. \\
& +4 \sum_{m=1}^{\infty} \sum_{n=1}^{\infty}(-1)^{n} I_{m}(\alpha) I_{n}(\alpha) \\
& \left.\times \int_{0}^{\phi_{l}} \int_{\phi}^{\phi_{l}} \cos m \phi \cos n x d x d \phi\right]
\end{aligned}
$$


where

$$
\begin{aligned}
\int_{0}^{\phi_{l}} \int_{\phi}^{\phi_{l}} \cos m \phi \cos n x d x d \phi= \\
\quad=\left\{\begin{array}{c}
\left.\cos (n-m) \phi_{l}\left[\frac{1}{n m}+\frac{1}{n(n-m)}\right]\right) \text { when } n \neq m \\
-\frac{4 \cos n \phi_{l}}{n m}-\frac{1}{(n-m) n}
\end{array}\right) \\
=\left(\frac{1}{m^{2}}-\frac{\cos m \phi_{l}}{m^{2}}\right) \quad \text { when } n=m
\end{aligned}
$$

This expression may be computed without the aid of a large-scale digital computer because the sequence $I_{n}(\alpha)$, and consequently the above series, converges quite rapidly.

However, the most important result which we seek can be obtained in closed form. This is the frequency of skipping cycles, or, in other words, the inverse of the expected time between skipping cycles, which is $T\left(\phi_{l}=2 \pi\right)$. It is clear from Eq. (63) that when $\phi_{l}=2 \pi$

$$
T(2 \pi)=\frac{2 \pi^{2}}{\gamma} I_{0}^{2}(\alpha)=\frac{\pi^{2} \alpha I_{0}^{2}(\alpha)}{2 B_{L}}
$$

where we have used

$$
\gamma=\frac{N_{0} K^{\prime}}{4}=\frac{A K}{\alpha}=\frac{4 B_{L}}{\alpha}
$$

so that

frequency of skipping cycles $=\left(2 B_{L}\right) /\left[\pi^{2} \alpha I_{0}^{2}(\alpha)\right]$

This parameter normalized by $B_{L}$ is shown as a function of $\alpha$ in Fig. 10.

For large SNR, $\alpha$,

$$
I_{0}(\alpha) \sim\left(e^{\alpha}\right) /(2 \pi \alpha)^{1 / 2}
$$

so that

frequency of skipping cycles $\simeq\left[\left(4 B_{L}\right) / \pi\right] e^{-2 \alpha}$ for large $\alpha$.

Another parameter which is equally significant is the frequency of dropping or advancing half cycles $\left(\phi_{l}=\pi\right)$.

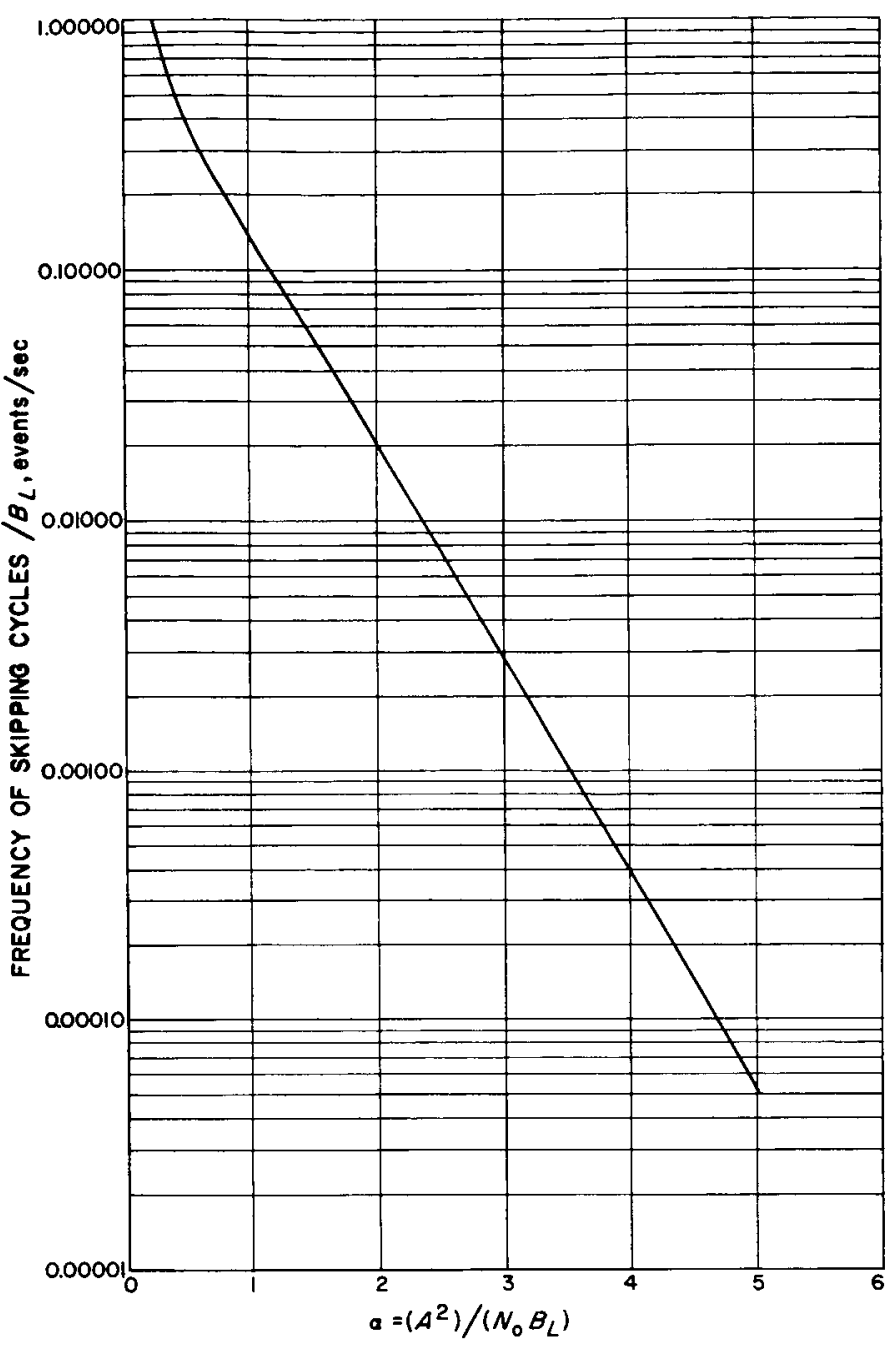

Fig. 10. Frequency of skipping cycles normalized by loop bandwidth for first-order loop where $\omega=\omega_{0}$

In the mechanical analog this corresponds to the pendulum arriving at the unstable equilibrium position and returning to the stable equilibrium position, either by the same route or by going around the full revolution. It is nearly intuitive that for a Markov process the frequency of this event is exactly double the frequency of skipping cycles. However, to show this rigorously, we note that the expected time for the pendulum to go from the equilibrium position $\phi=0$ to $\phi=\pi$ and to return is $T(\pi)+T^{\prime}(\pi)$, where $T(\pi)$ is the expected time to go from 0 to $\pm \pi$ and $T^{\prime}(\pi)$ is the expected time to go from $\pi$ to either 0 or $2 \pi . T(\pi)$ is given by Eq. (61) with $\phi_{l}=\pi$, while we can show that

$$
T^{\prime}(\pi)=\frac{1}{\gamma} \int_{0}^{\phi_{l}} \int_{0}^{\phi} \exp \alpha(\cos \phi-\cos x) d x d \phi
$$


The integrand is the same as that for $T(\pi)$, but the domain of integration is its complement with respect to the square of side $\pi$ (Fig. 11). Therefore,

$$
\begin{aligned}
T(\pi)+T^{\prime}(\pi) & =\frac{1}{\gamma} \int_{0}^{\pi} \int_{0}^{\pi} \exp \alpha(\cos \phi-\cos x) d x d \phi \\
& =\left(\pi^{2} / \gamma\right) I_{0}^{2}(\alpha)=[T(2 \pi) / 2]
\end{aligned}
$$

and

$$
\text { frequency of skipping half-cycles }=\left(4 B_{L}\right) /\left[\pi^{2} \alpha I_{0}^{2}(\alpha)\right]
$$

We can show that these results are approximately correct also for the second-order loop when $\alpha$ is large or $a<<1$ by means of the arguments used in Part $V$ to obtain Eq. (43) through (49).

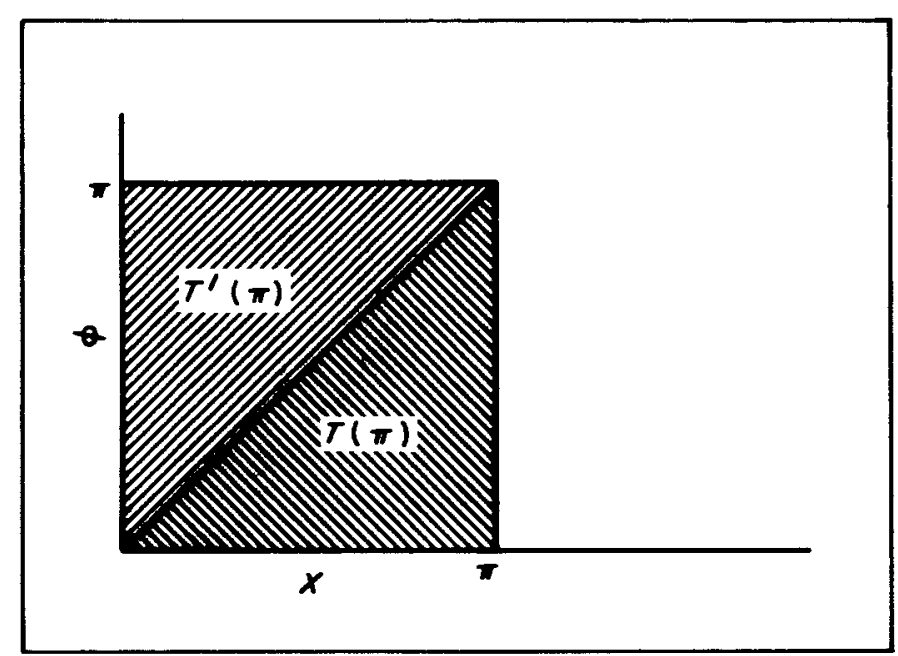

Fig. 11. Domains of integration for $T(\pi)$ and $T^{\prime}(\pi)$

\section{THRESHOLD CONSIDERATIONS AND CONCLUSIONS}

By means of the approximate models discussed in Part I, Van Trees (Ref. 5) and Develet (Ref. 6) have attempted to determine the threshold of the phase-locked loop; the threshold, as they define it, is the value of SNR for which the variance of the phase error becomes unbounded. However, we have shown by an exact analysis that the variance of the steady-state error is always bounded by the variance of the rectangular distribution $p(\phi)=(1 / 2 \pi)$ for $-\pi<\phi<\pi$, which equals $\left(\pi^{2} / 3\right)$. This is due to the fact that phase is measured to the nearest cycle (i.e., modulo $2 \pi$ ). On the other hand, if we count a change of a full cycle of phase as a phase error of $2 \pi$, and consider the resulting distribution and its variance, we find that the variance is unbounded for all noise densities greater than zero, for with this premise, the steady-state probability density has been shown to be a periodic function, so that its variance is necessarily unbounded. In simple physical terms, for any nonzero noise power, if the noise has a Gaussian distribution with probability one the loop will gain or lose a cycle if enough time elapses. (For the first-order loop, the expected time was obtained exactly in Eq. 64). Therefore, in the steady state (i.e., after an infinite interval of time has elapsed), the number of cycles skipped has a flat 
probability distribution; hence the probability density function is periodic. Since the idea of infinite variance for all finite SNR is ridiculous, we have no alternative but to accept the concept that phase is meaningful only modulo $2 \pi$, so that the variance is never unbounded. The mechanical analog of the simple pendulum discussed in Part II is useful in visualizing these conclusions.

If we redefine threshold to mean that value of SNR for which the linear model, or some other approximate model, becomes inadequate for the analysis, then our foregoing results can be utilized to determine the threshold of the model. It has been shown that, for a first-order loop with no frequency offset, the steady-state phase error has probability density

$$
p(\phi)=\frac{\exp (\alpha \cos \phi)}{2 \pi I_{10}(\alpha)} \quad-\pi \leq \phi \leq \pi
$$

where $\alpha=\left(A^{2}\right) /\left(N_{0} B_{L}\right)$ and that this is approximately correct also for a second-order loop with small integrator gain. Also, we have shown that the variance of $\phi$ in this case is given by

$$
\sigma_{\bar{\phi}}^{*}=\frac{\pi^{2}}{3}+4 \sum_{n=1}^{\infty} \frac{(-1)^{n} I_{n}(\alpha)}{n^{2} I_{0}(\alpha)}
$$

This is shown in Fig. 12 as a function of $1 / \alpha=N_{0} B_{L} / A^{2}$, where it is compared with the variance obtained from the linear model which is simply

$$
\sigma_{\phi}^{2}=\frac{N_{0} B_{L}}{A^{2}}=\frac{1}{\alpha} \quad(\text { Linear })
$$

Also shown in Fig. 12 are the results using the approximate models of Van Trees and Develet. Van Trees (Ref. 5) shows that for the first-order loop with no frequency offset (in our terminology)

$$
\sigma_{\bar{\phi}}^{\curvearrowright}=\frac{1}{\alpha-1} \quad(\text { Van Trees })
$$

so that the model yields an unbounded variance at $\alpha \leq 1$. Develet (Ref. 6) used the quasi-linearization technique of Booton (Ref. 7) which replaces the sinusoidal nonlinearity of Fig. 4 by its average gain, assuming that the input distribution is nearly Gaussian. The gain of a sinusoidal nonlinearity for an input of value $x$ is $A \cos x$. Therefore, the average gain when the input is Gaussian of mean zero and variance $\sigma^{2}$ is

$$
\int_{-\infty}^{\infty} A \cos x \exp \left(\frac{x^{2}}{2 \sigma^{2}}\right) d x=A \exp \left(-\frac{\sigma^{2}}{2}\right)
$$

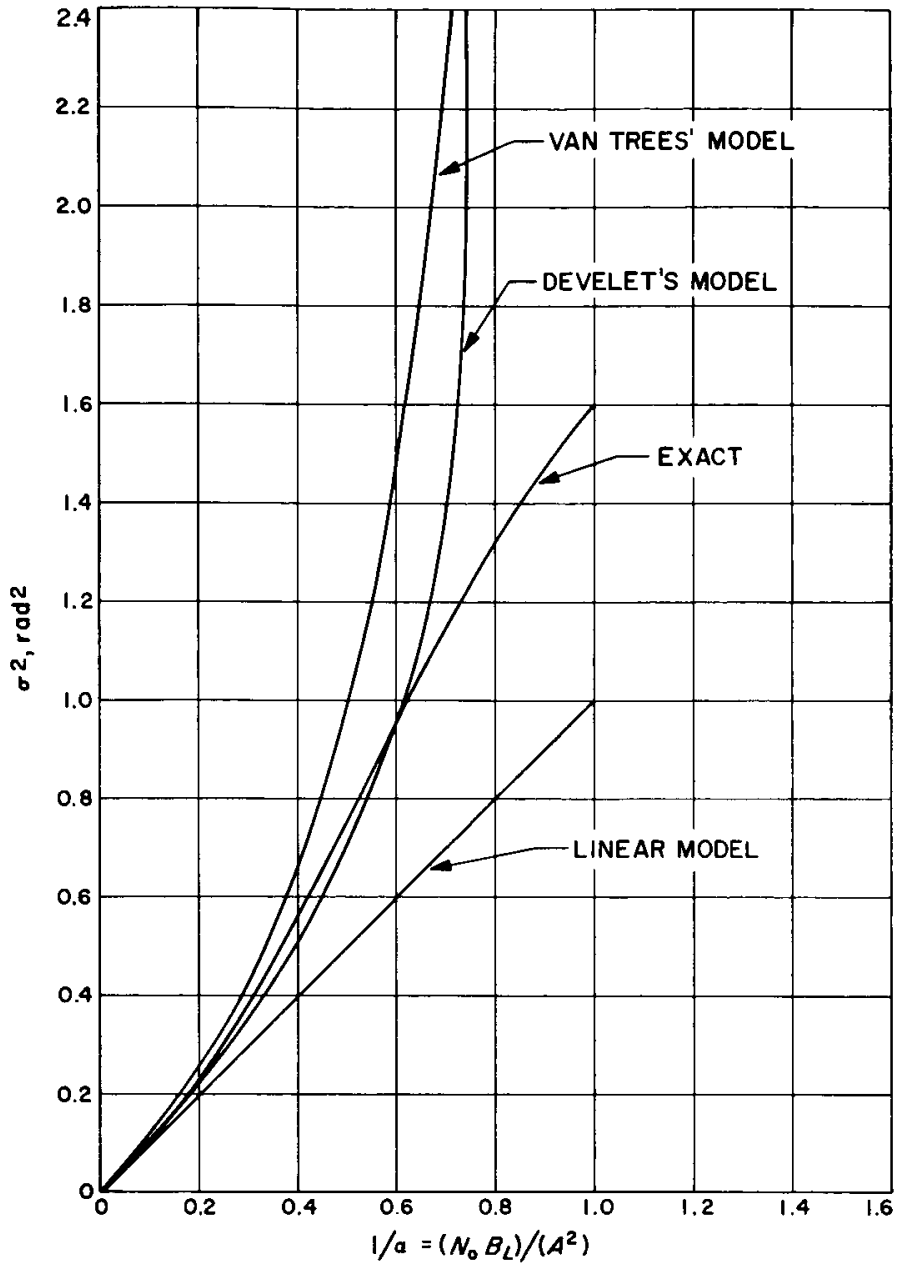

Fig. 12. Comparison of variance for first-order loop with results of approximate models

Replacing the nonlinear element of Fig. 4 by this gain, we obtain, by the usual linear analysis, the variance of the phase error for the first-order loop:

$$
\sigma^{2}=(1 / \alpha) \exp \left(\sigma^{2} / 2\right) \quad(\text { Develet })
$$

The solution of this transcendental equation yields the value of the variance which is also shown in Fig. 12. The maximum of $\sigma^{2} \exp -\left(\sigma^{2} / 2\right)$ is $2 / e$ so that there can be no solution for $\alpha<e / 2$. This is the point of unbounded variance for Develet's model.

In Fig. 12 we see that for SNR $=\alpha>1$, the linear model always yields an underestimate while Van Trees' result is always an overestimate of the variance of the phase error, as might be expected from the nature of the approximations. With the linear model the error 
in the approximation is less than $20 \%$ as long as $\left[\left(N_{0} B_{L}\right) / A^{2}\right]<1 / 4$, or $\alpha>4$ which is a figure often quoted by experimenters as a threshold of the linear model. Develet's model yields by far the most accurate result. In fact, it differs from the exact result by less than $10 \%$ for $\left[\left(N_{0} B_{L}\right) / A^{2}\right]<0.65$, or $\alpha>1.54$. Thus it would appear that if the exact solution cannot be obtained, as is the case for higher order loops, colored noise, or a modulated carrier, the Booton quasi-linearized model would yield quite accurate results when the SNR in the loop bandwidth is above 1.5 .

The other significant result of this Report, which can be used to define threshold, is the frequency of skipping cycles. It was shown in Part VI that for a first-order loop with no frequency offset or a second-order loop with very small integrator gain, the

$$
\text { frequency of skipping cycles }=\left(2 B_{L}\right) /\left[\pi^{2} \alpha I_{0}^{2}(\alpha)\right]
$$

which is plotted in Fig. 10. Thus we might set the threshold of the system as the SNR below which the frequency of skipping cycles exceeds a given value. Thus, for example, let $B_{L}=20 \mathrm{cps}$ and the maximum allowable frequency of skipping cycles be once every minute. Then we see from Fig. 10 that the threshold of the system is at $\alpha=3.6$. Note, however, that when the system operates at $\alpha=7.2,3 \mathrm{db}$ above threshold, the frequency of skipping cycles drops to once every $20 \mathrm{hr}$ reflecting the exponential behavior of this expression. This definition of threshold is most significant for coherent tracking applications wherein the doppler frequency is measured and integrated to obtain relative range information. Loss or gain of a cycle will yield incorrect results.

A third definition of threshold is the SNR for which the absolute value of the loop phase error $|\phi|$ exceeds a given value $\phi_{0}$ exactly half the time. For the first-order loop with $\omega=\omega_{0}$, this information is available from the cumulative probability distribution of Fig. 6. For example, if we set $\phi_{0}$ at $\pi / 4 \mathrm{rad}$, we find from Fig. 6 that the SNR at which $|\phi|$ exceeds this value exactly half the time is $\alpha=1.1$. We see also that when the SNR is $3 \mathrm{db}$ above this threshold level (i.e., $\alpha=2.2$ ), then $|\phi|$ exceeds $\pi / 4$ rad only about three-tenths of the time.

It is felt that the third definition of threshold has the least significance. The definition in terms of skipping cycles is most meaningful for ranging and tracking applications. However, the most useful result is the determination of the threshold of validity of the various models of the phase-locked loop. By comparing the variance of the phase error computed from each of the models with the actual variance for the first-order loop, which is the only case for which an exact solution in closed form is available, we have been able to determine these validity thresholds. The linear model underestimates the variance by less than $20 \%$, for SNR $>4(6 \mathrm{db})$. Van Trees' model overestimates $\sigma^{2}$ to within this accuracy for SNR $>2.5$ (4 db), while Develet's model is accurate to within $10 \%$ for SNR $>1.54(1.7 \mathrm{db})$. It does not necessarily follow that each model will yield equal accuracy for more complicated loops or signals, but these figures do represent lower bounds on the over-all validity. They also provide a ranking on the merits of the three models. It appears that the simpler the expression for variance, the less accurate is the result. If we wish merely to obtain bounds on performance, the results of Fig. 12 suggest that we use the linear model as an upper bound and Van Trees' model as a lower bound. On the other hand, if we are willing to solve a transcendental equation of the type of Eq. (69), it appears that Develet's model produces significantly greater accuracy over a much wider range of SNR. 


\section{REFERENCES}

1. Davenport, W. B., Jr., and Root, W. L., Random Signals and Noise, McGraw-Hill Book Co., Inc., N. Y., 1958.

2. Gruen, W. J., "Theory of A. F. C. Synchronization," Proceedings of the Institute of Radio Engineers, Vol. 41, No. 8, August 1953, pp. 1043-8.

3. Viterbi, A. J., "Acquisition and Tracking Behavior of Phase-Locked Loops," Proceedings of Symposium on Active Networks and Feedback Systems, Vol. X, Polytechnic Institute of Brooklyn, Brooklyn, April 1960, pp. 583-619.

4. Jaffe, R. M., and Rechtin, E., "Design and Performance of Phase-Lock Circuits Capable of Near-Optimum Performance Over a Wide Range of Input Signal and Noise Levels," Institute of Radio Engineers Transactions on Information Theory, Vol. IT-1, No. 1, March 1955, pp. 66-76.

5. Van Trees, H. L., A Threshold Theory for Phase-Locked Loops, Lincoln Laboratory Technical Report No. 246, Massachusetts Institute of Technology, Lexington, Mass., August 22, 1961.

6. Develet, J. A., Jr., "A Threshold Criterion for Phase-Lock Demodulation," Proceedings of the Institute of Radio Engineers, Vol. 51, No. 2, February 1963, pp. 349-356.

7. Booton, R. C., Jr., "The Analysis of Nonlinear Control Systems with Random Inputs," Proceedings of Symposium on Nonlinear Circuit Analysis, Polytechnic Institute of Brooklyn, Brooklyn, April 1953, pp. 369-391.

8. Margolis, S. G., "The Response of a Phase-Locked Loop to a Sinusoid Plus Noise," Institute of Radio Engineers Transactions on Information Theory, Vol. IT-3, March 1957, pp. 135-144.

9. Tikhonov, V. I., "The Effect of Noise on Phase-Lock Oscillation Operation," Automatika i Telemekhanika, Vol. 22, No. 9, 1959.

10. Tikhonov, V. I., "Phase-Lock Automatic Frequency Control Application in the Presence of Noise," Automatika i Telemekhanika, Vol. 23, No. 3, 1960.

11. Uhlenbeck, G. E., and Ornstein, L. S., "On the Theory of Brownian Motion," The Physical Review, Vol. 36, September 1930, pp. 823-841.

12. Wang, M. C., and Uhlenback, G. E., "On the Theory of Brownian Motion II," Reviews of Modern Physics, Vol. 17, No. 2 and 3, April-July 1945, pp. 323-342.

13. Andronov, A. A., Pontryagin, L. S., and Witt, A. A., "On the Statistical Investigation of a Dynamical System," Journal of Experimental and Theoretical Physics, Vol. 3, 1933, p. 165.

14. Siegert, A. J. F., "On the First Passage Time Probability Problem," The Physical Review, Vol. 81, No. 4, 1951.

\section{ACKNOWLEDGMENT}

The writer is indebted to Prof. J. N. Franklin and Dr. E. C. Posner for several valuable discussions during the preparation of this manuscript. 